\title{
OBSERVATIONS TECHNIQUES-FERMENTATIVES SUR L'EMPLOI D'UN LEVAIN DE STR. FAECALIS ET SUR L'IMPORTANCE DE CES TENDANCES DE CROISSANCE MESOPHILES \\ DANS LA FABRICATION DES FROMAGES A PATE DURE CUITE PREPARES A BASE DE LAIT CRU PAUVRE EN GERMES
}

\author{
par \\ Joseph A. KURMANN \\ Professeur et chef des laboratoires. \\ Ecole de Laiterie de Grangeneuve-Fribourg, Suisse. \\ Laboratoire de Biotechnologie Laitiere.
}

\section{I. - Introduction}

Pour la fabrication de fromage à base de lait cru pauvre en germes (Gruyère, Emmental) il importerait de connaître l'avantage de l'emploi de levains mésophiles. Il ne se pose pas la même question pour les fromages fabriqués à base de lait pasteurisé, puisqu'on a pris l'habitude d'ajouter toujours des levains mésophiles composés de Str. lactis cultivés à différentes températures $\left(30^{\circ}\right.$ et $18^{\circ} \mathrm{C}$, soit uniquement à $30^{\circ} \mathrm{C}$ ou à $22^{\circ} \mathrm{C}$, culture de beurrerie) $[5,8]$. Ces levains ont pour rôle : 1) d'empêcher ou de ralentir le développement des producteurs de gaz à des températures inférieures où les bactéries thermophiles ne sont plus en mesure de le faire (avant la coagulation et éventuellement après le pressage), 2) de ralentir le développement d'autres germes indésirés, 3) éventuellement de favoriser la fermentation lactique.

Nous connaissons deux sortes de bactéries mésophiles qui sont capables de se développer et de jouer un rôle utile dans le lait et le fromage : 1. les streptocoques lactiques mésophiles (str. lactis et str. cremoris) ; 2. les streptocoques fécaux avec leurs tendances de croissance mésophile (str. faecalis).

Vu que différents essais préliminaires, où fut employé une culture mésophile de Str. lactis dans la fabrication de pâtes dures cuites, n'ont pas été concluants [7], nous nous sommes attachés à l'emploi d'un levain d'enterocoques composé de Str. faecalis. Ces bactéries ont une tendance de croissance mésophile et thermophile. Pour notre usage la tendance de croissance mésophile nous intéresse spécialement, c'est-à-dire la possibilité de l'employer comme un levain mésophile (cultivé à $25^{\circ} \mathrm{C}$ ) pour combattre différents défauts de fromages (d'ouverture, d'arôme, de pâte, de fermentation lactique). 


\section{II. - Généralités sur l'ensemencement du lait avec des bactéries mésophiles d'un lait cru pauvre en germes}

\section{1) Importance pour les fabrications à base de lait pasteurisé.}

Pour la fabrication de pâtes dures cuites à base de lait pasteurisé (Gruyère pasteurisé, etc.), on ajoute toujours comme déjà mentionné, des levains mésophiles travaillant à différentes températures. Ceci montre l'importance que l'on attache aux bactéries mésophiles pour la fabrication de ces fromages. On peut donc, par analogie, déduire, comme ce fût déjà exprimé par $P$. Ritter [9], que les bactéries mésophiles ont aussi leur rôle à jouer dans les fromages à pâte dure cuite fabriqués à partir de lait cru.

\section{2) Infection du lait cru en bactéries mésophiles et nombre de germes totaux.}

L'infection du lait cru en bactéries mésophiles est de plus en plus variable et déficiente en fonction de la diminution du nombre de germes totaux dans le lait. En Suisse actuellement on trouve relativement peu de streptocoques lactiques dans le lait de fabrication. Par contre on rencontre souvent des streptocoques fécaux (str. faecalis, etc.). La nécessité d'ajouter des levains mésophiles varie d'une fromagerie à l'autre selon l'infection en bactéries mésophiles (str. lactis, str. faecalis, etc.). Dans un lait trop riche en germes, les cultures ajoutées peuvent difficilement accomplir leur fonction. Les mauvaises bactéries se trouvent en trop grand nombre. Leur activité ne peut plus être stoppée ou supprimée par les bactéries mésophiles. L'adjonction d'une culture mésophile produit done plus souvent un meilleur effet dans un lait cru pauvre en germes (entre $20000-100000$, jusqu'à éventuellement 300000 et plus de germes par ml).

\section{3) La température favorable de développement au cours de la fabrication.}

Les bactéries thermophiles ont leur température favorable de développement entre 28-30 et $49^{\circ} \mathrm{C}$. Cet intervalle de température, nous le trouvons uniquement à partir du moment où le lait est réchauffé pour la coagulation soit jusqu'à 24 heures après la fabrication.

Les bactéries mésophiles ont des possibilités de développement dans le lait, avant et au cours de la coagulation, jusqu'au chauffage et ensuite dans le fromage refroidi à $30-35^{\circ} \mathrm{C}$ (environ 16 heures après la fabrication) jusqu'à ce qu'il ait pris la température de cave $\left(15-18^{\circ} \mathrm{C}\right)$. Les entérocoques (Str. faecalis), par leur tendance de croissance thermophile, peuvent se développer aussi au-dessus de $40^{\circ} \mathrm{C}$ et résistent beaucoup mieux aux hautes températures que les streptocoques lactiques mésophiles. 


\section{4) Choix de l'espèce bactérienne.}

a) Streptocoques lactiques mésophiles (Str. lactis et cremoris).

Si l'adjonction de levains mésophiles de Str. lactis ou cremoris, s'avère concluante pour la fabrication avec du lait pasteurisé, ce n'est pas nécessairement le cas pour les fabrications avec du lait cru. Comme il s'agit pour le lait pasteurisé d'un lait extrêmement pauvre en germes (avec peu d'espèces différentes), les streptocoques lactiques mésophiles ont de bonnes possibilités de développement. Ils se trouvent pratiquement seuls. Par contre, dans le lait cru, on trouve une flore plus riche et plus variée en bactéries antagonistes. L'antagonisme des bactéries mésophiles est trop faible (trop vite épuisé) pour stopper le développement de germes indésirables. Par exemple, dans les pâtes molles fabriquées avec du lait pasteurisé nous avons presque toujours un développement de colibacilles et un gonflement limité du fromage [11].

Toutefois nous ne voudrions pas exclure la possibilité que dans certaines conditions favorables les streptocoques lactiques mésophiles pourraient aussi apporter une amélioration de la qualité du fromage à différents points de vue.

b) Str. faecalis (enterocoques ou streptocoques fécaux).

Il nous semble que Str. faecalis a des avantages sur Str. lactis et cremoris pour l'emploi en fromagerie. Les streptocoques fécaux sont des germes particulièrement vivants, et ils résistent à des conditions de vie défavorables. Par exemple d'après Buttiaux [12] :

1. Résistent dans les milieux extérieurs et aux différents traitements appliqués aux aliments.

2. Survivent à un chauffage à $60-65^{\circ} \mathrm{C}$ pendant $30-45$ minutes.

3. Se développent entre 10-45-500 C (tendances de croissance psychrophiles, mésophiles et thermophiles).

4. Sont beaucoup moins sensibles au froid etjà la pénicilline que la plupart des autres bactéries en laiterie.

5. Se multiplient en présence de 6,5 p. 100 de sel.

6. Se multiplient en présence de 40 p. 100 de bile.

7. Supportent de fortes variations de $p \mathrm{H}$.

8. Dans la majorité des aliments, se multiplient activement grâce à leurs caractères particuliers.

9. Présentent une tolérance relative au chlore, etc.

En conclusion, c'est done Str. faecalis qui se développe le mieux parmi les germes mésophiles avant, pendant et après la fabrication, c'est-à-dire aux diverses températures. En comparaison avec les streptocoques fécaux, les streptocoques lactiques mésophiles (Str. lactis et cremoris) sont des germes faibles et peu résistants. De ce fait, il 
résulte que le bon développement des streptocoques lactiques mésophiles (qui n'est pas toujours assuré) dépend : 1) largement de la flore du lait, 2) du milieu de culture (présence d'inhibiteurs de croissance, etc. et 3 ) de la résistance des souches aux conditions de vie défavorable.

On peut donc conclure que les streptocoques fécaux résistent le mieux aux conditions de vie défavorables parmi les germes non sporulés que l'on trouve en laiterie.

\section{III. - Facteurs qui influencent l'infection du lait de chaudière en streptocoques fécaux}

\section{1) Stockage du lait.}

L'adjonction de culture de Str. faecalis suppose toujours un stockage correct du lait à la ferme et à la laiterie. L'effet variable dans l'emploi de la culture provient en partie du traitement variable du lait chez le producteur et à la laiterie. Le développement des streptocoques fécaux est influencé par : 1) le refroidissement (ou non refroidissement) chez le producteur, 2) la température du lait pendant le transport et la durée de transport, 3) le refroidissement à la fromagerie, 4) la vitesse de refroidissement et 5) le temps de stockage du lait.

\section{a) Stockage du lait entre 10 et $20^{\circ} \mathrm{C}$.}

Actuellement en Suisse le lait pour la fabrication des fromages est stocké entre $10 / 12$ et $18 / 20^{\circ} \mathrm{C}$.

Plus la température de stockage est élevée, plus fort est le développement des mauvais germes du groupe coli-aérogènes, mais aussi celui des streptocoques fécaux. Au-dessus de $20^{\circ} \mathrm{C}$ les streptocoques thermophiles peuvent aussi se multiplier.

\section{b) Stockage du lait $\grave{a} 5$ et $7^{\circ} C$.}

Si un jour on est équipé dans les fromageries suisses pour refroidir le lait à 5-70 $\mathrm{C}$, la nécessité d'ajouter des cultures de str. faecalis pourrait éventuellement s'accroître, car en dessous de $10^{\circ} \mathrm{C}$ les streptocoques fécaux ne se développent plus, mais il se multiplient au-dessus de $10^{\circ} \mathrm{C}$. Dans les fromageries avec une infection faible en str. fécaux et où le stockage du lait au dessous de $10^{\circ} \mathrm{C}$ ne permet plus un enrichissement, on pourrait éventuellement (dans certains cas) améliorer la qualité des fromages par une adjonction de culture de str. fécaux. 


\section{Infection du lait.}

On constate dans la production d'un lait cru pauvre en germes que les changements dans l'infection sont fréquents (groupe coliaérogènes, microcoques, str. fécaux, str. lactiques mésophiles, etc.).

Les streptocoques fécaux habitent presque tous les intestins humains et animaux. On les rencontre parfois, pourtant, dans les produits n'ayant pas été soumis à des souillures fécales, au moins apparentes [12].

\section{a) Ustensiles.}

Comme déjà mentionné on trouve dans les ustensiles à lait un nombre variable de streptocoques fécaux, qui sont résistants aux procédés de nettoyage. L'infection en str. fécaux est fortement influencée par le procédé de lavage appliqué; 1) par le rinçage, 2) par la nature du produit de nettoyage employé, 3) par la température de l'eau chaude ou le procédé de stérilisation.

Le nombre de streptocoques fécaux que l'on trouve dans les ustensiles bien lavés est souvent assez faible, mais il ne faut pas oublier que ceux-ci peuvent s'enrichir pendant le stockage du lait au-dessus de $10^{\circ} \mathrm{C}$.

b) Air.

L'air de l'étable peut être infecté en streptocoques fécaux, notamment par les particules d'excréments. Ailleurs, les streptocoques fécaux se trouvent presque toujours dans l'air des exploitations d'industries alimentaires et infectent souvent les matières alimentaires fabriquées (poudre de lait, etc.) [12].

\section{c) Infection de la mamelle et l'hygiène de la traite.}

On peut aussi trouver des streptocoques fécaux dans la mamelle. L'infection varie généralement de quelques centaines à quelques milliers de germes par $\mathrm{ml}$.

Comme les streptocoques fécaux sont particulièrement nombreux dans les excréments, le lait est variablement infecté, selon la propreté de la traite, celle des vaches, etc.

\section{d) Les cultures empiriques de fromageries.}

Non seulement dans les cultures pures, mais encore bien plus souvent dans les cultures empiriques sur du sérum (petit-lait) on trouve des streptocoques fécaux. Les cultures qui dégagent de fortes odeurs caséeuses sont infectées par ces bactéries. On peut donc ajouter par les cultures de fromageries (présures, petit-lait, etc.) de ces bactéries. D'une façon générale on peut dire, que les streptocoques fécaux sont des germes d'infection les plus fréquents des levains et des cultures-de fromagerie. 


\section{3) Circuit d'infection.}

L'infection ainsi que la quantité de culture de streptocoques fécaux à verser sont déterminées par le cycle d'infection :

a) Le grand cycle d'infection.

Dans le grand cycle d'infection le petit-lait est retourné aux producteurs dans leurs ustensiles, ce qui permet toujours de réinfecter le lait (fig. 1a).

\section{b) Le petit cycle d'infection.}

Ce circuit d'infection est limité aux ustensiles de la laiterie, qui ont contact avec le lait de fabrication, et sur les cultures empiriques préparées à partir du sérum (pris pendant la fabrication du fromage). (Fig. 1b).

\section{IV. - Préparation et repiquage des cultures de Str. faecalis}

\section{1) Préparation.}

Dans le lait de mélange la flore des streptocoques fécaux se laisse souvent enrichir par une simple incubation (par exemple prélèvement de $40 \mathrm{cl}$ de lait dans un tube de réductase) dans un thermostat à $25^{\circ} \mathrm{C}$. La coagulation du lait doit s'effectuer après 16-24 heures. Cet enrichissement peut être contrôlé par un ensemencement sur le milieu. L'enterococcus-Agar Difco N. 746-01, incube à $39^{\circ} \mathrm{C}$. L'enrichissement est douteux lorsque l'on constate une forte production de gaz (bulles de gaz dans le lait caillé, qui provient le plus souvent d'Oospora lactis. De cet enrichissement on isole des souches dont on étudie le pouvoir acidifiant, l'activité au test du bleu de méthylène [6], la production de substances d'arômes par dégustation et, éventuellement, si l'hémolyse est négative. Comme il existe aussi des souches qui fermentent faiblement le lactose, il faut choisir uniquement les souches qui attaquent fortement ce sucre. Si l'on recherche une amélioration de l'arôme, il faut noter que Storgards et Hieteranta [4] ont trouvé que ce ne sont pas toutes les souches qui favorisent l'arôme.

Si l'on ne dispose pas de laboratoire pour faire ce travail et si l'on cherche uniquement à améliorer l'arôme, on peut éventuellement faire un essai avec la culture de Str. durans (ni protéolytique, ni hémolytique, il produit faiblement de l'acide lactique) fourni par Chr. Hansens Laboratoires A/S, (Sct. Annae Plads 3, Kobenhaven K. Danmark). Cette culture de str. durans est employée pour la fabrication du cheddar dans le but d'améliorer l'arôme [10]. Il faut toutefois noter qu'elle n'a pas les mêmes propriétés que notre levain de Str. faecalis. 


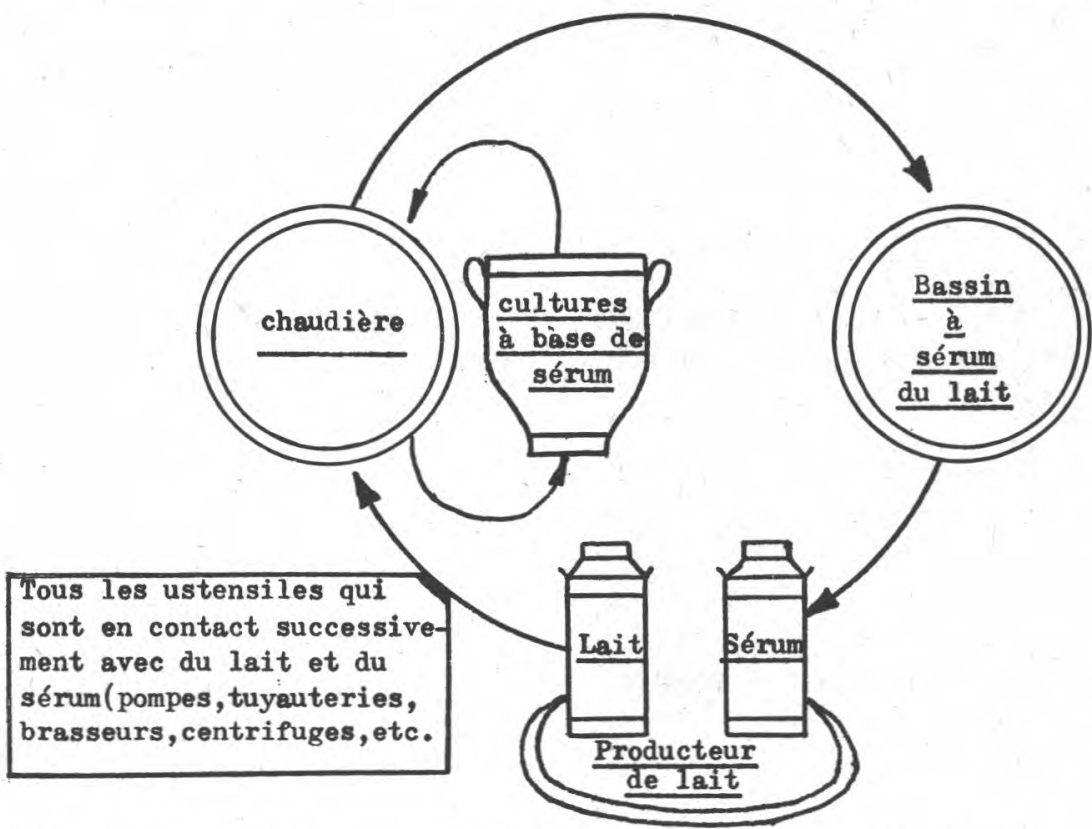

FIG. 1-a. - Grand circuit d'infection en streptocoques fécaux lorsque le sérum est rendu aux producteurs dans les ustensiles à lait.

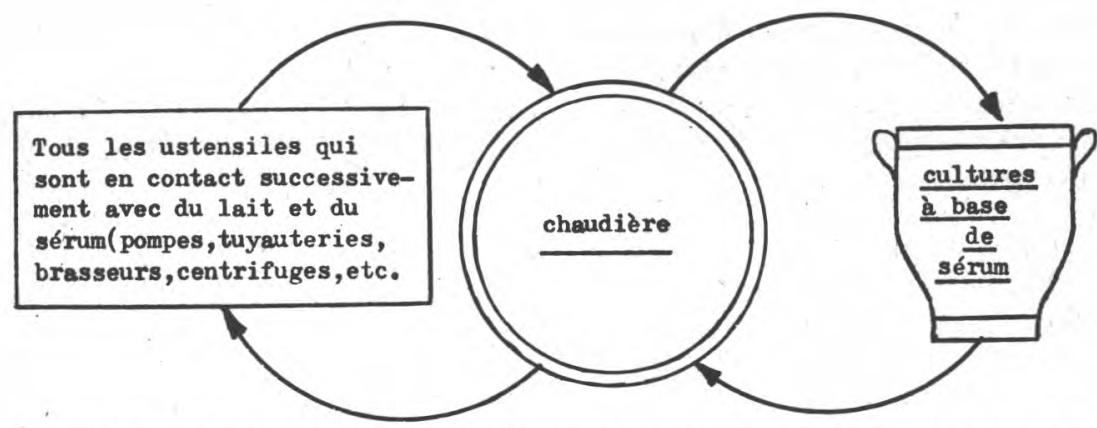

FIg. 1-b. - Petit circuit d'infection en streptocoques fécaux dans la fromagerie où le sérum n'est pas rendu aux producteurs dans les ustensiles à lait.

\section{2) Repiquage.}

Nous recommandons de repiquer la culture sur du lait bouilli et de préparer ainsi chaque jour la quantité de levain nécessaire, qui est extrêmement faible en raison de la petite quantité d'ensemencement. 
Le repiquage des cultures peut se faire dans le cas le plus simple en prélevant du lait de chaudière ou du sérum (petit-lait), après l'ensemencement avec le levain de str. faecalis, qui est ensuite enrichi par une incubation durant 16-24 heures à $25^{\circ} \mathrm{C}$. Cela est évidemment un procédé de repiquage qui demande une certaine expérience.

\section{3) Ensemencement du lait de chaudière et CRègles pour l'emploi des cultures de str. faecalis.}

a) Un stockage du lait approprié est supposé.

L'adjonction de culture de Str. faecalis suppose toujours un stockage correct du lait à la ferme et à la laiterie. Nos essais ont été faits sur un mélange de lait du soir, refroidi à $12-15^{\circ} \mathrm{C}$ et de lait du matin, non refroidi. On fabriquait le matin comme on travaille actuellement le lait de fromagerie en Suisse.

b) Quelques règles pour l'emploi de la culture.

Quelques règles pour l'emploi de la culture sont énumérées ci-après :

- La quantité de cultures de streptocoques fécaux à verser doit être beaucoup plus adaptée que celle des autres cultures aux conditions microbiologiques de chaque exploitation. Celle-ci doit concorder et s'harmoniser avec les cultures de ferments lactiques ajoutées. Le développement des ferments lactiques ne doit pas être entravé et la quantité optimale de la culture doit être trouvée pour chaque sorte de fromages.

En fonction de la grande vitalité et du développement à une échelle beaucoup plus vaste de température que les autres microbes, on travaille. avec des faibles quantités. L'ensemencement est fait à raison d'environ $40 \mathrm{~cm}^{3}$ de culture pour 1000 litres de lait.

- On doit veiller à ce que le sérum (petit-lait en Suisse) utilisé pour la préparation des cultures en fromagerie ne soit pas trop infecté en streptocoques fécaux de façon que les bactéries lactiques puissent se développer normalement. Ce danger ne se présente pas dans l'emploi de cultures sélectionnées (pures), qui sont repiquées sur du lait pasteurisé.

- Dans le cas ou le sérum (petit-lait) n'est pas retourné aux producteurs dans les ustensiles à lait on peut éventuellement verser d'avantage de cultures.

- Toutefois, l'emploi de la culture nécessite une surveillance plus stricte de la fabrication que l'utilisation des autres cultures. 


\section{c) Préensemencement.}

Pour faciliter le développement, on chauffe souvent le lait avant la coagulation à $25^{\circ} \mathrm{C}$. Le temps de l'ensemencement avant l'emprésurage est prolongé et raccourci en fonction du développement des bactéries fécales (par exemple 1/2-1 h).

Quelquefois on ajoute au lait refroidi, stocké entre 12-17 ${ }^{\circ} \mathrm{C}$, ces cultures de Str. faecalis pour peupler le lait. Ce procédé doit être bien surveillé (à cause d'une suracidification du lait). Il est à noter que les streptocoques fécaux se développent très lentement à $10^{\circ} \mathrm{C}$ et ils coagulent le lait après environ $5-10$ jours.

\section{d) Infection alternative.}

$\mathrm{Vu}$ leur résistance aux différents procédés de lavage et de nettoyage (survie d'une plus grande quantité de streptocoques fécaux par rapport aux autres germes), on obtient rapidement dans certaines exploitations ( par les ustensiles à lait, par le petit circuit de l'infection, mais davantage avec le grand circuit de l'infection, etc.) une infection suffisante du lait en streptocoques fécaux. De ce fait, on doit pratiquer dans certaines exploitations une infection non continue, alternative c'est-à-dire un ensemencement seulement tous les 3 ou 6 jours par exemple.

\section{4) Abaissement de la qualité du fromage par une infection trop forte.}

Presque tous les microbes sont nuisibles s'ils se trouvent en trop grand nombre dans le lait. Beaucoup de perturbations actuelles dans la fabrication des fromages à pâte dure cuite proviennent d'un développement excessif des streptocoques fécaux.

L'ensemencement trop conséquent en streptococcus faecalis diminue la qualité du fromage. Des observations sont aussi confirmées par certains essais de Winkler [13], qui a travaillé avec de grandes quantités de cultures $(0,25,0,5,1,0$ et 2,0 p. 100). Un ensemencement trop fort provoque;

- Le plus souvent une suracidification du lait et du fromage (pâte trop acide et trop fine ; lainure) ;

- Une mauvaise ouverture. Ceci est dû probablement à un développement retardé des bactéries lactiques thermophiles (streptococcus thermophilus ?);

- Perturbation d'acidification. Winkler a observé dans le fromage d'Emmental, une acidification plutôt retardée. Il est possible qu'une trop grande quantité de streptocoques fécaux inhibe ou ralentisse la croissance des ferments lactiques. Lorsqu'on ensemence différentes quantités de streptocoques fécaux avec les bactéries lactiques thermophiles l'antagonisme éventuel devrait 
être étudié. Il en résulte de ce fait que les cultures empiriques préparées à base de sérum (petit-lait) perdent de leur vitalité et que les streptocoques fécaux s'enrichissent, ce qui peut être aussi néfaste à la fabrication ;

- D'après Winkler l'arôme du fromage d'Emmental fabriqué serait aussi influençable (c'est-à-dire moins bon) ;

\section{5) Facteurs qui influencent l'effet variable observé par diffé- rents auteurs dans l'emploi de la culture.}

L'effet dans l'emploi de la culture de Str. faecalis pour différentes sortes de fromages et selon les observations différentes de plusieurs auteurs $[1,10,13]$ est variable :

a) Si on ne recherche pas une qualité supérieure du fromage le résultat n'est pas facilement positif, car il s'agit des améliorations qui sont peut-être à peine perceptibles lorsqu'on fabrique des fromages d'une qualité moyenne Lorsqu'on recherche une qualité supérieure on court beaucoup de risques, des incertitudes, et de ce fait la fabrication doit être beaucoup plus surveillée.

b) Dans quel sens une amélioration de la qualité du fromage est recherchée? Dans certains cas il est plus facile d'obtenir une amélioration que dans d'autres. Ceci dépend en grande partie de la flore et de la technique de fabrication qui varie fortement parmi les différentes sortes de fromages. Certaines améliorations de qualité peuvent être plus facilement obtenues avec une culture de Str. faecalis dans certaines variétés de fromage que dans d'autres.

c) Le résultat dépend de la flore du lait et de l'infection en bactéries fécales en général, comme aussi du genre de levain de-bactéries lactiques thermophiles ou mésophiles employé.

d) Il dépend aussi des souches choisies. Parmi les espèces employées en laiterie, Str. faecalis présente les plus fortes variations dans l'activité (par exemple, le pouvoir de fermenter le lactose et de former l'arôme peuvent être très différentes d'une souche à l'autre).

e) La quantité de la culture de Str. faecalis à verser doit concorder et s'harmoniser apec la quantité de cultures de ferments lactiques ajoutées. Le développement des ferments lactiques ne doit pas être entravé et la quantité optimale de la culture de Str. faecalis à verser doit être trouvée pour chaque sorte de fromages.

\section{V. - Observations sur les fromages fabriqués et les défauts que l'on peut combattre}

L'emploi d'une culture de Str. faecalis permet avant tout de combattre des défauts provenant d'un manque d'infection en microbes mésophiles dans un lait cru pauvre en germes: empêcher la 
multiplication d'une mauvaise flore bactérienne provenant de l'infection du lait (groupe coli-aérogènes, microcoques, etc.). $D$ 'où il est recommandé de limiter l'emploi de la culture sur des perturbations de fabrication [1]. C'est ainsi un moyen de lutte nouveau et complémentaire pour éviter les défauts différents (ouverture, arôme, pâte, fermentation lactique) de fromages à pâte dure cuite en vue de la recherche d'une qualité supérieure de ces fromages (hautes exigences du point de vue ouverture, arôme ét pâte).

\section{a) Ouverture.}

Ces améliorations proviennent avant tout du fait que certains producteurs de gaz sont empêchés, dans le lait stocké ainsi qu'au début de la fabrication, de se développer (influence des tendances de croissance mésophiles).

Gruyère Suisse.

Nous avons eu dans certains cas du succès avec l'emploi de cette culture pour combattre le défaut " mille-trous" provoqué par un développement limité des producteurs de gaz dans le fromage. La culture fut introduite, 1 heure avant la coagulation du lait de chaudière ou encore beaucoup plutôt dans le lait refroidi à $12-14^{\circ} \mathrm{C}$ pendant son stockage $[2,3]$.

\section{Emmental.}

Pour les fromages d'Emmental, vu en Suisse l'exigence extrême au point de vue ouverture (régularité de la répartition, grandeurs et nombre des trous), la culture a permis d'obtenir en certain cas une meilleure ouverture (moins chargée, plus régulière).

Ces améliorations proviennent avant tout du fait que certains producteurs de gaz sont dans le lait stocké comme aussi au début de la fabrication, empêchés de se développer (par les tendances de croissance mésophiles).

\section{b) Arôme.}

Dans certaines meules de fromage d'Emmental il est possible d'améliorer l'arôme (goût plus pur), ce qui est probablement dû à l'inhibition de la croissance de microbes nuisibles par Str. faecalis (surtout dans le lait stocké). Pour certains cas ceci peut éventuellement aussi provenir de la forte dégradation de la caséine. Toutefois, il est aussi intéressant de noter que Str. faecalis est un très fort producteur de diacétyle et il est possible qu'il contribue de ce fait à la formation de l'arôme de certains fromages [14].

\section{c) Amélioration de la pâte.}

Comme les cultures de streptocoques lactiques en général ont une influence favorable sur la pâte on peut observer la même 
influence pour les streptocoques fécaux pour le fromage d'Emmental. L'influence sur la pâte mérite d'être encore mieux étudiée en raison des enzymes secrétées qui sont fortement protéolytiques.

\section{d) Fermentation lactique.}

Comme déjà mentionné pour la préparation de la culture on se sert uniquement de souches de Str. faecalis, qui fermentent activement le lactose. Par la tendance de croissance mésophile, Str. faecalis peut fermenter ce sucre à une température plus basse que les lactobacilles et les streptocoques thermophiles. La fermentation lactique par les tendances de croissance mésophile est d'une importance primordiale, par contre la fermentation par les tendances de croissance thermophiles est d'une importance secondaire.

Winkler [13] a trouvé en cultivant ensemble Str. faecalis et $L b$. helveticus en lait stérilisé, que l'acidification était meilleure. Par son pouvoir protéolytique, il est possible que Str. faecalis favorise le développement des lactobacilles. Par l'adjonction de culture de Str. faecalis on arrive parfois' à normaliser c'est-à-dire à abaisser un $p \mathrm{H}$ trop élevé dans le fromage d'Emmental âgé de 24 heures, mais il faut aussi remarquer que cet effet ne peut pas être généralisé dans tous les cas. $\mathrm{Vu}$ la diminution du $p \mathrm{H}$ dans le fromage âgé de 24 heures, se pose le problème de savoir si l'acidification déficiente provient d'un manque de développement de streptocoques thermophiles ou d'un manque d'activité de lactobacilles ou encore d'autres facteurs (composition du milieu de culture, etc).

\section{VI. - Conclusions}

1) L'utilité des streptocoques fécaux (avec ses différentes tendances de croissance) dans la fabrication de fromage à base de lait cru pauvre en germes est le mieux démontrée par le fait, dans l'exemple précédent, qu'il est possible dans certains cas de combattre avec certitude le défaut "mille-trous". Ceci est assez nouveau dans la microbiologie des fromages à pâte dure cuite fabriqués à base de lait cru et est causé par un changement d'infection du lait cru (moins de germes totaux par rapport à une quantité quelquefois considérable de germes indésirés). Toutefois, on peut parler d'une utilité limitée des cultures de Str. faecalis dans la fabrication des fromages.

2) L'effet variable qu'on observe au cours de l'emploi de la culture dans les différentes fromageries provient de diverses causes, qui sont valables de façon générale pour l'infection du lait en str. fécaux, comme aussi en bactéries indésirées :

a) Traitement très variable du lait (refroidissement ou non refroidissement chez le producteur); 
b) Hygiène de la traite et infection (en excréments) variable du lait;

c) Différents modes de réfrigération et de stockage à la laiterie ;

d) Cycle d'infection (petite ou grande);

e) Lavage et stérilisation très variables des ustensiles.

Ces variations peuvent donc parfois neutraliser le bon effet provenant de l'adjonction de la culture.

3) Les streptocoques fécaux sont donc un groupe de bactéries qui jouent un certain rôle dans la microbiologie des fromages [10]. Ce rôle est assez difficile à démontrer :

a) Du fait de la variation de l'infection naturelle en str. fécaux comme déjà mentionné ;

b) Du fait de la forte variation de la flore indésirable dans un lait cru pauvre en germes;

c) Du fait qu'une présence trop importante de ces germes est plutôt nuisible à la qualité du fromage ;

d) Du fait que ces germes se trouvent numériquement en nombre inférieur par rapport aux bactéries lactiques ;

e) Du fait que leurs enzymes secrétés sont particulièrement actifs (exemple : protéases, etc.).

\section{Résumé}

L'utilité de Streptococcus faecalis dans la fabrication du fromage à pâte dure est démontrée par le fait que les germes de ce groupe peuvent dans certains cas combattre le défaut " mille trous ». Cette utilité est cependant limitée et elle dépend, en particulier, de la teneur initiale du lait en germes et du degré des contaminations.

\section{Sum mary}

The utility of Streptococcus faecalis in the making of hard cheese is evident since the germs of this group can in some cases cope with the defect called " a thousand holes ». Yet this utility is limited, and it depends particularly on the initial bacterial content of the milk and on the degree of pollutions. 


\section{BIBLIOGRAPHIE}

[1] J.A. KURmann. - Versuche zur Vorreifung der Käsereimilch in schlecht fabrizierenden Emmentaler-und Greyerzerkäsereibetrieben mit einer mesophilen Kultur von str. faecalis. Deutsche MolkereiZeitung, 84, 1732-1734, 1963.

[2] J. A. Kurmann. - Le défaut "mille-trous " du fromage, ses causes et les moyens employés pour le combattre. Le Technicien du tait, $n^{\circ} 209$ (1965).

[3] J. A. KurmanN. - Untersuchungen und Beobachtungen im Zusammenhang mit den Käselochungsfehler "Tausendlöchler " oder " milletrous ». Versuchsdurchführung mit Greyerzerkäsen. Deutsche Molkere $\dot{i}$-Zeitung, Kempten, 84, 1364-1366, 1963.

[4] T. Storgards, M. Hieteranta et J. Heikinheimo. - Voir dans Manuale Lactis, II. Folge, vol, 5, p. 423.

T. Storgards et M. Hieteranta. - Voir Manuale Lactis, III. Folge, vol. 5, p. 423.

[5] A. M. GUfrRault. - La fromagerie devant les techniques nouvelles. Ed. Sep, Paris, 1956, p. 279.

[6] J. A. KuRManN. - Die Methylenblaureduktaseprobe, ein einfacher Aktivitätstest für die Herstellung und Untersuchung von Streptokokkensäureweckern in der Käsefabrikation. Deutsche MolkereiZeitung, 87, 324-325, 1966.

[7] a. H. Stettler. - Schweiz. Milchutg. Nos 63 et 64, p. 385-386, 1961.

b. J. Andres et O. Bolliger. - Schweiz. Milchzeitung. No 8, p. 37 et 41-43, 1963.

c. Observations personnelles.

[8] J. A. Kurmann. - Die Herstellung von französischem Greyerzerkäse aus past. Milch. Molkerei- und Käserei-Zeitung, 12, 66, 1961.

[9] P. Ritter. - Voir Schweiz. Milchztg. No 64, p. 394, 1961.

[10] V. F. Kosikowski et G. Mocquot. - Progrès de la technologie du fromage. Etudes Agricoles FAO. No 38, p. 138-139, 1958.

[11] a. J. A. Kurmann. - Ueber die schwedische Käsebereitung. Schweiz. Milchztg. No 28, p. 177, 1959.

b. J. A. Kurmann. - Mikrobiologische Untersuchungen bei der Reifung des past. St.-Paulin-Käses. Schweiz. Milchztg. No 29, Wiss. Beil. $\mathrm{n}^{0} 45$ (1957).

[12] R. Butriaux. - Les cocci a Gram positif des aliments, interprétation de leurs présence au point de vue de l'hygiène. Annales de l'Institut Pasteur de Lille, 13, 179-185, 1962.

[13] S. WINkLer. - Züchtungs- und Käsereiversuche mit Sc. faecalis. Milchwissenschaftliche Berichte, 4, 219-240, 1954.

[14] National Institute for Research in Dairying at Shinfield in Reading. Annual Report. Ref. Le Lait, 23, 44, 1943. 